W3 North: Far-Infrared and Radio Molecular Observations

Harley A. Thronson, Jr.,

P. R. Schwartz, Howard A. Smith, Charles J. Lada, W. Glaccum, and D.A. Harper

\title{
LaRhM COPY
}

21984

MANGLEY RESEARCH CENTER LIBRARY, NASA

May 1984 HAMPTON VIRGINIA

\section{N/Sก}

National Aeronautics and Space Administration

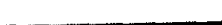




\section{W3 North: Far-Infrared and Radio Molecular Observations}

Harley A. Thronson, Jr., University of Wyoming

P. R. Schwartz

Howard A. Smith, E. O. Hulburt Center for Space Research, Naval Research Laboratory

Charles J. Lada, Stewart Observatory, University of Arizona

W. Glaccum

D. A. Harper, Yerkes Observatory, University of Chicago

\section{N/SA}

National Aeronautics and

Space Administration 
Ap. J., 284, in press

W3 NORTH: FAR-INFRARED AND RADIO MOLECUL.AR OBSERVATIONS

\author{
Harley A. Thronson, Jr., ${ }^{1}$ P. R. Schwartz, 2 \\ Howard A. Smith, ${ }^{2}$ Charles J. Lada, 3,4 \\ W. Glaccum, ${ }^{5}$ and D. A. Harper ${ }^{5}$
}

Received 27 December 1983

1 Wyoming Infrared Observatory, University of Wyoming.

2 E. 0. Hulburt Center for Space Research, Naval Research Laboratory.

3 Steward Observatory, University of Arizona.

4 Alfred P. SToan Foundation Fellow.

5 Yerkes Observatory, University of Chicago. 


\begin{abstract}
We report the results of far-infrared and radio molecular co observations of the W3 North star-forming region. The object shows extended dust and gas emission, which we interpret as the result of fairly advanced disruption of a molecular cloud. This idea is supported by an estimate for the age of the embedded $H$ II region. It is possible that W3 North is the oldest object among the W3 complex of sources.
\end{abstract}

Subject headings: infrared: sources -- interstellar: molecules -nebulae: H II regions -- nebulae: individuat (W3) 


\section{INTRODUCTION}

The W3 North $(G 133.8+1.4)$ source is the northernmost member of a string of active star-forming regions that marks the western boundary of the giant $H$ II region W4. Although not often a specific target of observational programs, W3N has been included in a number of surveys of the region. At far-infrared wavelengths Thronson, Campbe11, and Hoffmann (1980) found bright, extended emission from the region, although they were not able to clearly delineate between internal and external heating. Thronson and Price (1982) discussed the large-scale mid-infrared structure of the region, within which W3N appeared as a shoulder at the edge of the bright W3A source. Lada et al. (1978) presented a ${ }^{12}$ Co survey of the area at an angular resolution of $2: 6$ and 8'. These observations clearly place W3N in a ridge of molecular emission that includes the three other bright infrared sources, W3A, W3 $(\mathrm{OH})$, and AFGL 333. Lada et a1. argue that these four sources were formed out of material compressed by the expansion of W4. Dickel (1980) proposed an al ternative model for the source.

The only infrared observations with an angular resolution sufficient to study structure in the source was that of Zeilik (1979), although only a $2.2 \mu \mathrm{m}$ map was presented. At this wavelength he found fairly weak emission, extended by about 1' and offset slightly from the radio continuum peak. Fazio et al. (1975) scanned close to the location of W $3 \mathrm{~N}$ and estimated a lower limit to its far-infrared flux density. Furniss et al. (1974) mapped much of the W3 complex and detected W3N, but used beam sizes of $4^{\prime}-5^{\prime}$ which made it difficult to analyze the object in any detail. 
Radio continuum surveys of the area (Rohlfs, Braunsfurth, and Hills 1977; Wendker and Altenhoff 1977) show W3N as a distinct source, but it takes angular resolution better than a few arcmin to fully separate the many sources in the region (Sullivan and Downes 1973, Schraml and Mezger 1969). These latter observations suggest that the object has structure on a scale size of a few arcmin and is powered by a star - or stars with a Tuminosity equivalent to an 07 zero age main sequence (ZAMS) star.

A usefur summary of the many observations of the $13 / W 4$ region has been presented by Dickel et al. (1980).

\section{THE OBSERVATIONS}

\section{a.) The Far-infrared Observations}

The observations reported here were obtained at the Nasmyth focus of the $0.9 \mathrm{~m}$ telescope onboard NASA's Kuiper Airborne Observatory during January, 1983. The detector system was a multi-filter, seven-element array configured with a central detector and six close-packed detectors surrounding it. The angular resolution of each of the seven apertures was $49^{\prime \prime}$ and the center-to-center separation between the middle and an outer aperture was $57^{\prime \prime}$. The reference beam was $7: 5$ to the nor theast of W3N. The objects used for calibration were W3(OH) and OMC 1.

The source was observed in four broad passbands. It was first mapped at $60 \mathrm{\mu m}$ (Fig. 1). The multi-channel array was then centered on the maximum emission and we obtained photometry with the three other filters. The results are presented in Table 1 . The flux density in the central beam is presented, along with the flux density measured for all seven channels. Only for the $60 \mu \mathrm{m}$ filter was there sufficient data to estimate a total flux density for the source. Inspection of Figure 1 
suggests that roughly $20 \%$ of the total $60 \mu \mathrm{m}$ flux density was missed by our mapping, particularly to the south of the peak.

The far-infrared luminosity was found for the source by assuming that all the filters had the same spatial distribution as that at $60 \mu \mathrm{m}$. No correction was made for possible emission outside the boundaries of the area mapped. The object was assumed to be at a distance of $2.3 \mathrm{kpc}$. The flux densities in the central channel at emission peak were used to estimate a dust temperature and emission optical depth. Following the usual convention for multi-filter far-infrared photometry, we assumed that the source spectrum was of the form $F_{v} \propto \nu B_{v}\left(T_{d}\right)$, where $T_{d}$ is the dust temperature. Although this spectral shape fits the data in Table 1, we do not have sufficient spectral resolution and coverage to distinguish between the function adopted here or other similar ones. [The problem of deriving source parameters from far-infrared photometry is considered more fully in Thronson (1985).] Fitting our observations by eye gives $T_{d}=75 \pm 15 \mathrm{~K}$, where the uncertainty is our estimate of the fit. An emission optical depth at $60 \mu \mathrm{m}$ was found for the peak using $F_{v}=\Omega B_{v}\left(T_{d}\right)(1-\exp [-60])$, where $\Omega$ is the beam solid angle. For the peak of $W 3 N$, we calculated $\tau_{60}=0.003$.

\section{b.) The Millimeter-wave Observations}

Mill imeter-wave emission from the $J=1 \rightarrow 0$ transition of co was observed in W3N during September, 1981 using the $11 \mathrm{~m}$ telescope of NRAO. ${ }^{6}$ The ${ }^{12} \mathrm{CO}$ emission was mapped over a $6^{\prime} \times 6^{\prime}$ area centered on the

${ }^{6}$ The National Radio Astronomy Observatory is operated by Associated Universities, Inc., under contract with the National Science Foundation. 
far-infrared maximum. A single ${ }^{13} \mathrm{CO}$ spectrum was obtained at the far-infrared maximum. The beamwidth was $66^{\prime \prime}$, the map was sampled every beam-width, and the reference beam position was $30^{\prime}$ to the east in an area devoid of C0 emission (Lada et a1. 1978). The frequency resolution for the observations reported here was $250 \mathrm{kHz}$. The calibration object was $W 3(O H)$ for which we took $T_{A}^{*}\left({ }^{12} \mathrm{CO}\right)=16.8 \mathrm{~K}$ and $T_{A}^{*}\left({ }^{13} \mathrm{CO}\right)=7.1 \mathrm{~K}$.

The radio molecular data was analyzed in the usual manner (e.g., Dickman 1978, Martin and Barrett 1978). The ${ }^{12} \mathrm{CO}$ emission was assumed to be optically thick and its calculated excitation temperature was assumed also to hold for ${ }^{13} \mathrm{CO}$. The ${ }^{13} \mathrm{CO}$ column density at the far-infrared maximum, $N\left({ }^{13} \mathrm{CO}\right)$, was found under the assumption of thermodynamic equilibrium. From inspection of our map of the distribution of $T_{A}^{*}\left({ }^{12} \mathrm{CO}\right)$, we estimated a forward beam coupling coefficient of 0.54 . This object is part of an extensive program of study of the CO emission from the $W 3$ region. The column density and mass distribution are discussed here, but the velocity structure analysis is deferred to a later paper (Thronson, Lada, and Hewagama 1984)

\section{ANALYSIS AND DISCUSSION}

\section{a.) Morphology and Energetics}

The luminosity estimated for the source from our far-infrared data, $7 \pm 1.4 \times 10^{4} \mathrm{~L}_{0}$, is only slightly below that estimated from radio continuum observations (Schraml and Mezger 1969, Sullivan and Downes 1973). As noted in the previous section, our observations may underestimate the Tuminosity by $20 \%$, so we consider the infrared and radio continuum data to be in good agreement. The W3N object is excited 
by a star -..- or stars -..- with a luminosity equivalent to an 07 ZAMS star. The agreenent between the luminosity determined from the infrared and radio observations means that there is very little dust mixed in with the ionizing gas and competing for the ionizing photons. This is a common situation for evolved diffuse $H$ II regions and is discussed further in $\S$ IIIC.

The infrared data reported here are also in generally good agreement with that found by other investigators, after correction for apparent beam-size effects. This means that surrounding the region mapped in Figure 1 may be an extended area of low-level emission. In addition, with W3A only $12^{\prime}$ to the southwest, there can be contamination in the large beams of other experiments. In a 4 ' beam, Furniss et al. (1974) estimated a far-infrared luminosity of $1 \times 10^{5} \mathrm{~L}_{\odot}$ (a11 observations have been corrected to distance of $2.3 \mathrm{kpc}$, where necessary), in good agreement with our data. However, Thronson, Campbe11, and Hoffmann (1980) found $L_{F I R}=1.3 \times 10^{5} L_{\theta}$ in a $12^{\prime}$ beam. This latter observation would be particularly susceptible to the bright emission from W3A.

other than for ${ }^{12} \mathrm{CO}$, the maximum emission at all wavelengths longward of visual is close to the same position, within the uncertainties of observation (2.2 $\mu \mathrm{m}$ and $12.6 \mu \mathrm{m}$ : Zeilik 1979; $1.4 \mathrm{GHz}$ continuum: Sullivan and Downes; $60 \mu \mathrm{m}$ : this work). There is very little information as to size or structure of the source from other farminfrared observations. Fazio et al. (1975) roughly estimated a diameter greater than 2', but both Furniss et al. and Thronson, Campbeil, and Hoffmann found the object unresolved to their large beans. Inspection of Figure 1 shows that $2^{\prime}$ is a good estimate of the 
far-infrared half-power size. This is much larger than the $2.2 \mu \mathrm{m}$ size of the object (Zeilik 1979), an expected result since the near-infrared emission is dominated by free-free emission from the ionized gas or by the very hottest dust. However, the far-infrared size is only slightly larger than that found for the radio continuum (Sullivan and Downes 1973). The distribution of $\mathrm{T}_{A}^{*}\left({ }^{12} \mathrm{CO}\right)$ (Figure 2) is much more extended than either the far-infrared or radio continuum, which is a fairly common situation. It is less common to find only a modest local maximum at the far-infrared peak, as is the case for W3N.

Our data, as well as that at other wavelengths, show that W3N can be described as a partially obscured, diffuse $H$ II region. Visual photographs (e.g., Sullivan and Downes; Thronson, Campbell, and Hoffmann; Dickel et al.) clearly give the impression of ionized gas breaking through obscuring neutral material. The far-infrared and radio continuum observations show that the source has little compact, high brightness structure. The extended nature of $60 \mu \mathrm{m}$ emission suggests that the exciting $\operatorname{star}(s)$ in the object are not heavily dust enshrouded, but instead the stellar and nebular radiation is escaping to heat a large area of surrounding material. The fairly low molecular gas temperatures (Table 1), even at the far-infrared peak, is further evidence for more extended, diffuse structure in the source.

\section{b) Mass and Density}

Because the dust temperature is so high (Table 1) and the flux density is only moderately high, the $60 \mathrm{um}$ emission optical is low. This supports our description of W3N as a diffuse $H$ II region in which the typical far-infrared-emitting grain is embedded in a more intense radiation field than would be the case for a dense, compact $H$ II region. 
The low emission optical depth is reflected in the visual appearance of the source, as well as the modest ${ }^{13} \mathrm{CO}$ column density, $N\left({ }^{13} \mathrm{CO}\right)=7 \mathrm{x}$ $10^{15} \mathrm{~cm}^{-2}$. Using the relations in Dickman (1978), this is equivalent to 3 visual magnitudes of extinction. This level of obscuration, although low, is sufficient so that most of the visual and ultraviolet photons from the source will be absorbed by dust and re-radiated at infrared wavelengths. This is necessary to explain the agreement between the luminosity determined from the radio continuum observations and from the infrared data.

As Figure 2 shows, the molecular cloud associated directly with W3N is not clearly separated from more extensive material in the area. Therefore, only a rough estimate of the mass of the molecular material specifically associated with the infrared source can be made. If we take the W3N cloud to be $3^{\prime}(2 \mathrm{pc})$ in diameter, the relationship between mass and ${ }^{13} \mathrm{CO}$ column density found by Dickman results in a cloud mass of $230 M_{0}$. This assumes that the ${ }^{13} \mathrm{CO}$ column density at the infrared peak is characteristic of the rest of the source as we11, which is unlikely.

It is generally assumed that in most molecular clouds near active star-forming regions, the gas is heated by collisions with dust. We therefore expect that the material that dominates the far-infrared emission is mixed with that dominating the co emission. The $60 \mu \mathrm{m}$ optical depth should be related to the ${ }^{13}$ co column density. From our data at the infrared maximum we find $\sigma_{60}=4 \times 10^{-19} N\left({ }^{13} \mathrm{CO}\right)=$ $0.001 \mathrm{~A}_{v}$. This result is in good agreement with similar calculations by a number of investigators (see discussion in Thronson et al. 1984).

A fundamental parameter for any star-forming region is the volume density and we use the column density to calculate it for W3N. If the 
object is $2 \mathrm{pc}$ in diameter and we use $\mathrm{N}\left(\mathrm{H}_{2}\right) / \mathrm{N}\left({ }^{13} \mathrm{CO}\right)=5 \times 10^{5}$ from Dickman, we calculated $\mathrm{n}_{H_{2}} \approx 600 \mathrm{~cm}^{-3}$ from the co column density. This fairly low level is consistent with our picture of the source as a diffuse H II region.

\section{c.) The Age of W3 North}

It is the visual appearance of the object (Lada et al; Thronson, Campbe11, and Hoffmann) that most quickly gives the impression that this young, star-forming region has substantially disrupted its surrounding neutral cloud. The object shows extensive, visually bright emission centered very close to the infrared and radio continuum maximum. The fact that the $60 \mu \mathrm{m}$ and ${ }^{12} \mathrm{CO}$ emission are both very extended further supports the idea that the cloud is being dissipated.

As part of an extensive study of the evolution of the W3 system (see Thronson, Lada, and Hewagama for a more complete discussion), it is valuable to estimate an age for $W 3 N$. There is really only one technique suitable for this: deriving the expansion age of the H II region. This requires knowing initial conditions in $W 3 N$, but the uncertain values enter to a low power in the final expression. Spitzer (1978) derived equations describing the expansion of an $H$ II region, from which we may write its age

$$
t \approx \frac{4 r^{7 / 4}}{7 v} r_{s}^{-3 / 4}
$$

Here $r$ is the present radius of the ionized zone (0.6 pc, Harten 1976), $V$ is the expansion velocity $\left(\sim 10 \mathrm{~km} \mathrm{~s}^{-1}\right)$, and $r_{s}$ is the stromgren radius of the H II when expansion began (with $r_{s} \ll r$ ). This latter parameter may be replaced, using the standard expression for the Stromgren sphere. We therefore get

$$
t \approx \frac{4}{7}\left(\frac{4 \pi \alpha}{3 N_{l}^{-}}\right)^{1 / 4} \frac{r^{7 / 4}}{V} e^{1 / 2}
$$


where $\alpha$ is the hydrogen recombination coefficient, $N_{\ell}$ is the Lyman continuum flux for an $07 \mathrm{star}$, and $n_{e}$ is the electron density when the $\mathrm{H}$ II region was originally established. It is clear that $n_{e}$ is the most poorly known parameter, but on the basis of the most compact H II regions discussed in the literature, we adopt $n_{e} \sim 10^{5} \mathrm{~cm}^{-3}$. The resulting estimate for the age is $t \gtrsim 4 \times 10^{5}$ years. We consider this a weak lower limit since, of the parameters in the above equation most subject to change, $V$ may be lower than adopted here and $n_{e}$ may be larger.

Despite the uncertainties in $t$, it is worth noting that our estimate of the age of the HII region is very close to the age that Dickel (1980) estimates for the time that higher-density "clumps" (not stars) existed in the W3 region. In other words, the estimate for the $H$ II region that we derive here suggests that at least some stars in the complex were more evolved than in the Dickel scenario. 


\section{SUMMARY}

The luminous W3 North $H$ II region has been mapped at far-infrared wavelengths and in the emission of the $\mathrm{J}=1 \rightarrow 01$ ine of ${ }^{12} \mathrm{CO}$. We deduce the following characteristics about the source.

1. The object is excited by the equivalent of an 07 ZAMS star and is surrounded by extended $\mathrm{CO}$ and far-infrared emission.

2. Only a moderate-mass neutral cloud surrounds the H II region. Its density is in the range of $\mathrm{n}_{\mathrm{H}_{2}} \sim 600 \mathrm{~cm}^{-3}$ and appears to have an average visual extinction of $\sim 3$ mags.

3. The expansion age of the $H$ II region is $t \gtrsim 4 \times 10^{5}$ years. This is long enough to significantly disrupt the overlying material, which is exactly how the source appears in visual photographs. 
We appreciate the continued high level of support by the Ames Medium Altitude Missions Branch, and the air and ground crew of the KAO. We are also grateful for the traditionally fine support at NRAO. Mr. T Hewagama assisted in the analysis of the radio data and a helpful referee improved the presentation of this work. This work was supported by NASA grant NAG 2-134 and the University of Wyoming Division of Basic Research. 
TABLE 1

\section{SOURCE PARAMETERS}

Peak Position

$(60 \mathrm{~m})$

Flux Density (Jy)

Luminosity $(30 \mu m-200 \mu m)^{b}$

Peak Dust Temperature

Peak Gas Temperature

$40 \mu \mathrm{m}$
$60 \mu \mathrm{m}$
$100 \mu \mathrm{m}$
$160 \mu \mathrm{m}$

$b$

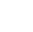




\section{REFERENCES}

Dicke1, H. R. 1980, Ap. J., 238, 829.

Dickel, H. R., Dickel, J. R., Wilson, W. J., and Werner, M. W. 1980, Ap. J., 237,711 .

Dickman, R. L. 1978, Ap. J. Supp1., 37, 407.

Fazio, G. G., Kleinmann, D. E., Noyes, R. W., Wright, E. L., Zeilik, M., and Low, F. J. 1975, Ap. J. (Letters), 199, L177.

Furniss, I., Jennings, R. E., and Moorwood, A. F. M. 1974, Proceedings of Eighth ESLAB Sumposium, ed. A.F.M. Moorwood, p. 61.

Harten, R. H. 1976, Astr. Ap., 46, 109.

Lada, C. J., Elmegreen, B. G., Cong, H., and Thaddeus, P. 1978, Ap. J. (Letters), 226, L39.

Martin, R. N., and Barrett, A. H. 1978, Ap. J. Supp., 36, 1.

Rohlfs, K., Barunsforth, E., and Hills, D. L. 1977, Astr. Ap. Supp., $30,369$.

Schraml, J., and Mezger, P. G. 1969, Ap. J., 156, 269.

Spitzer, L. 1978, Physical Processes in the Interstellar Medium (New York: John Wiley and Sons), p. 251.

Sullivan, W. T., and Downes, D. 1973, Astr. Ap., 29, 369.

Thronson, H. A. 1985, Ap. J., submitted.

Thronson, H. A., Campbel1, M. F., and Hoffmann, W. F. 1980, Ap. J., $239,533$.

Thronson, H. A., Lada, C. J., and Hewagama, T. 1984, Ap. J., submitted. 
Thronson, H. A., and Price, S. D. 1982, A. J., 87, 1288.

Thronson, H. A., Smith, H. A., Lada, C. J., Glaccum, W., Harper, D. A., Loewenstein, R. F., and Smith, J. 1983, M.N.R.A.S., submitted. Wendker, H. J., and A1 tenhoff, W. J. 1977, Astr. Ap., 43, 301. Zei1 ik, M. 1979, A. J., 84, 1566. 


\section{FIGURE CAPTIONS}

Fig. 1 - The distribution of $60 \mu \mathrm{m}$ flux density in 13 North. The contours are normalized to $1.00=910 \mathrm{Jy}$. The light outer boundary shows the extent of the area mapped. The beam size is represented by the cross-hatched circle. The cross is located at the emission maximum. The length of the four arms are each 15" long, the $2 \sigma$ uncertainty in absolute position.

Fig. 2 - The distribution of $\mathrm{J}=1 \rightarrow 0 \mathrm{~T}_{\mathrm{A}}^{*}{ }^{12} \mathrm{CO}$. The contours are labeled in kelvins. The beam size is represented by the cross-hatched circle. The cross is located at the position of maximum $60 \mu \mathrm{m}$ emission. The arms of the cross are each $15^{\prime \prime}$ in length, the pointing uncertainty of the $11 \mathrm{~m}$ telescope. 
ADDRESSES

W. Glaccum and D. A. Harper

Yerkes Observatory

Williams Bay, WI 53191

C. J. Lada

Steward Observatory

University of Arizona

Tucson, AZ 85721

P. R. Schwartz and H. A. Smith

E. 0. Hulburt Center for Space Research

Naval Research Lab.

Washington, D.C. 20375

H. A. Thronson

Department of Physics and Astronomy

University of Wyoming

Laramie, WY 82071 


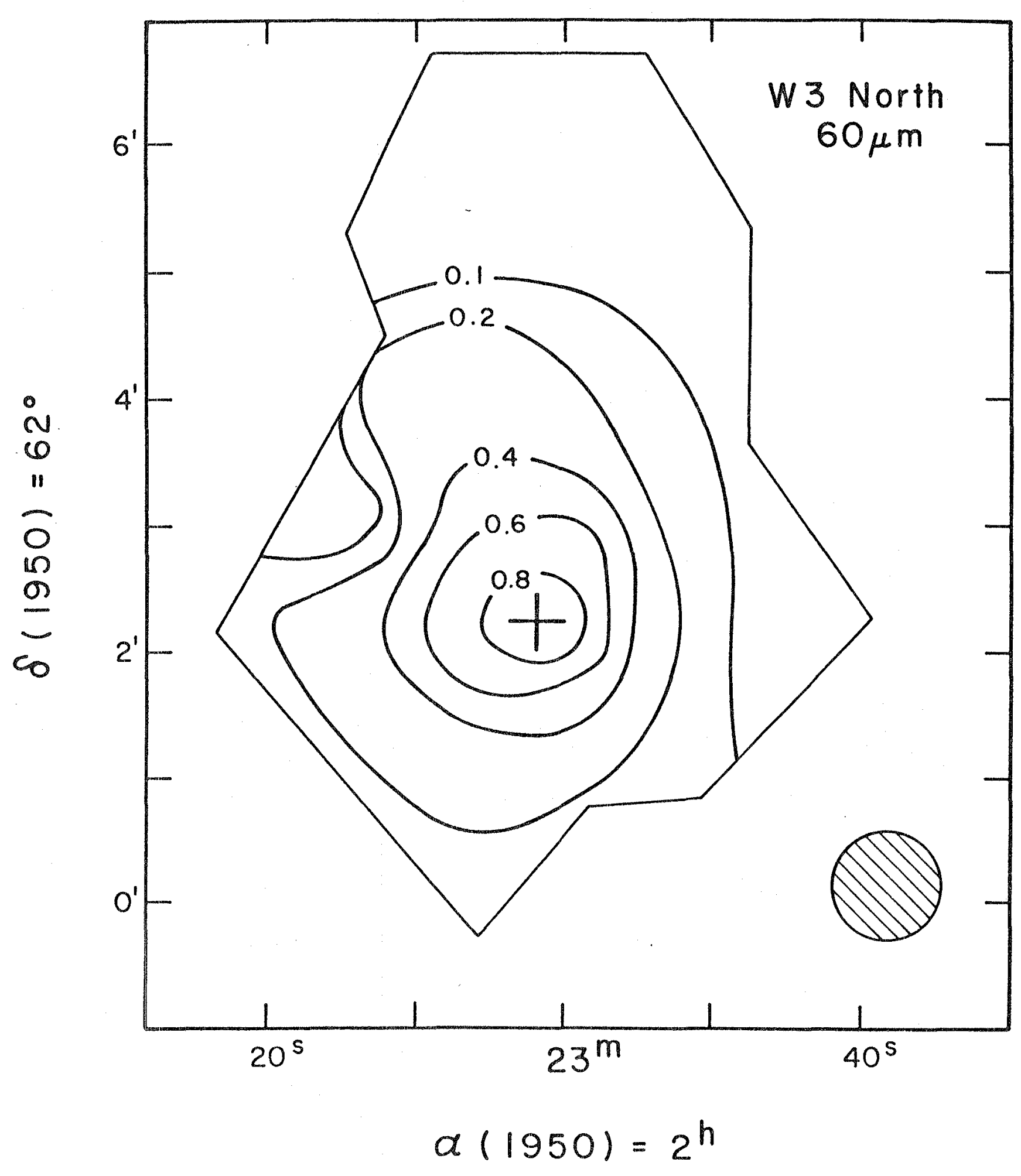

Fig. 1 


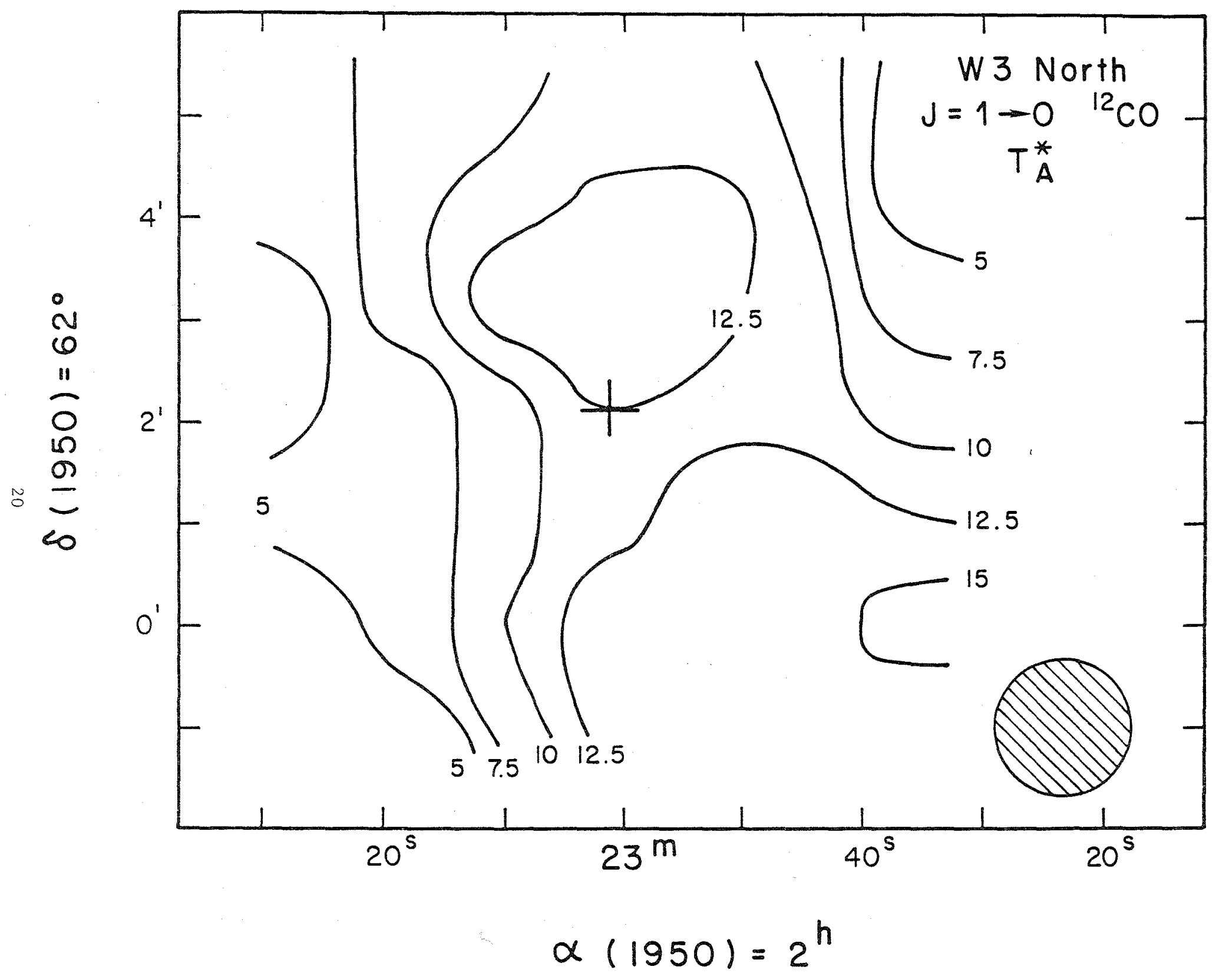

Fig. 2 


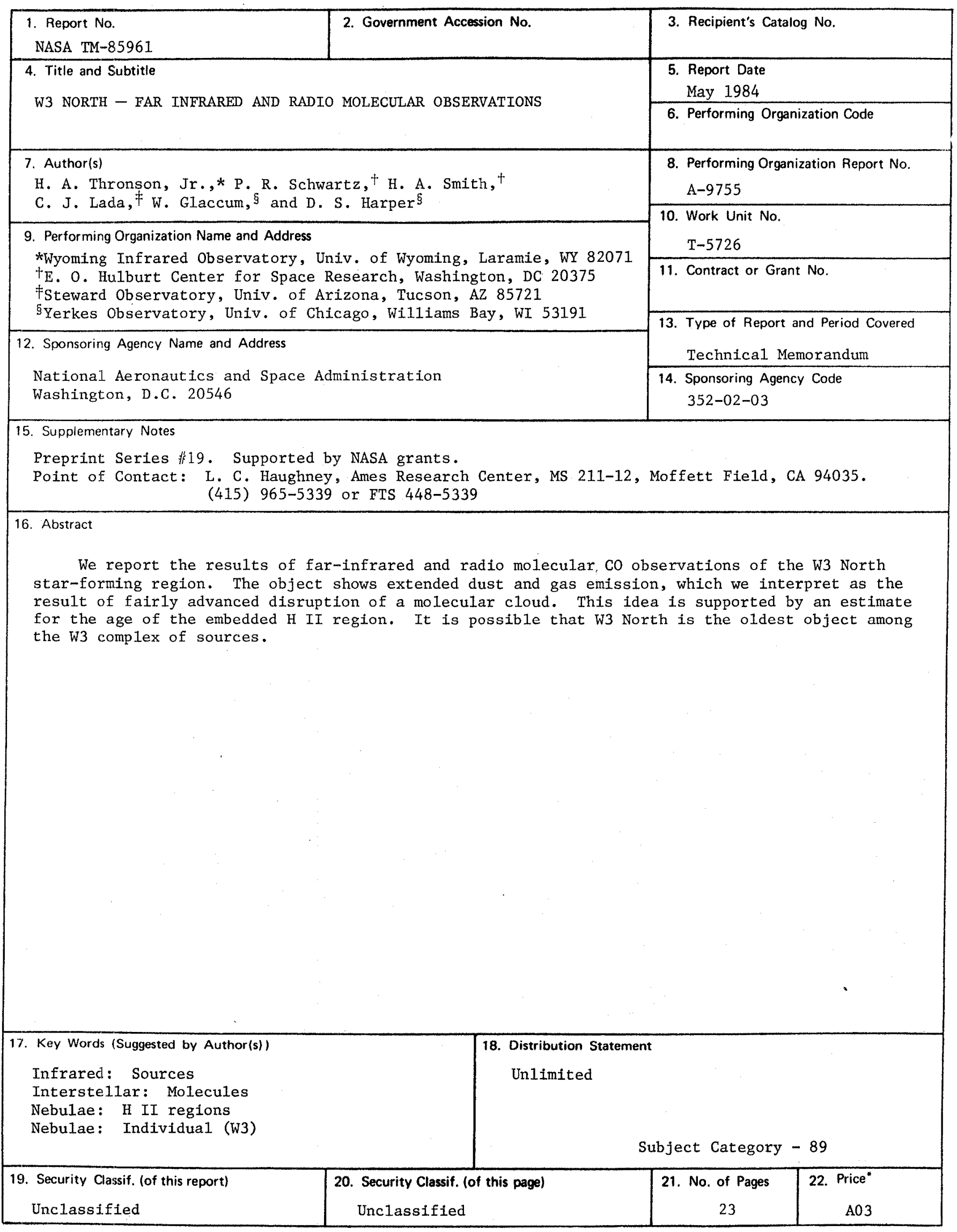


End of Document 